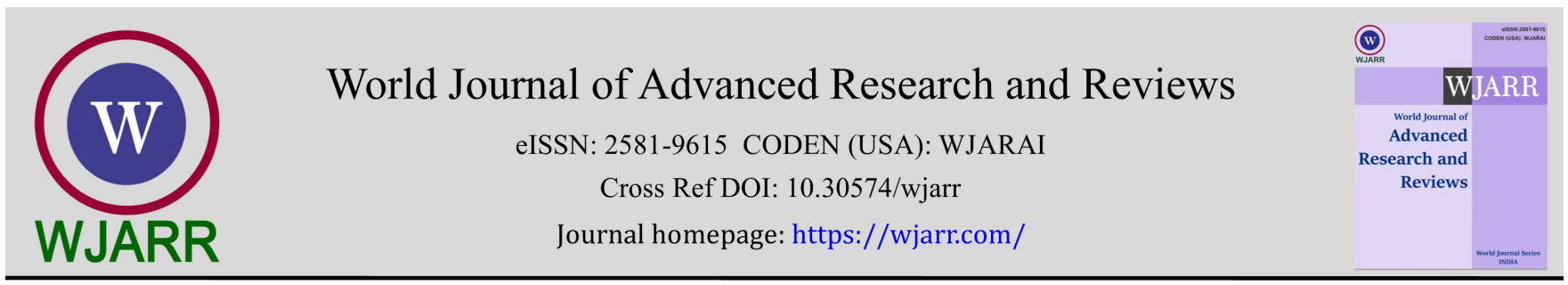

(RESEARCH ARTiClE)

Check for updates

\title{
The unnoticed truth: Patients on the heart transplantation waiting list are becoming cachexic
}

\author{
Ozlem Balcioglu 1, ${ }^{*}$ Ulvan Ozad ${ }^{2}$ and Umit Kahraman ${ }^{3}$ \\ ${ }^{1}$ Department of Cardiovascular Surgery, Faculty of Medicine, Near East University, TRNC Mersin 10 Turkey. \\ 2 Department of Plastic and Reconstructive Surgery, Faculty of Medicine, Near East University, TRNC, Mersin 10, Turkey. \\ 3 Department of Cardiovascular Surgery, Faculty of Medicine, Ege University, Turkey.
}

World Journal of Advanced Research and Reviews, 2021, 12(01), 225-229

Publication history: Received on 06 September 2021; revised on 09 October 2021; accepted on 11 October 2021

Article DOI: https://doi.org/10.30574/wjarr.2021.12.1.0516

\begin{abstract}
Heart transplantation is a major lifesaving surgery practiced globally on daily basis. The scarcity of donors has led to long waiting lists for the surgery. Both physiology and psychology of patients are affected severely during this period. The nutritional status is known to be correlated with postoperative outcomes. The aim of this study was to investigate the significance of the weight loss of patients by comparing their weights and ideal body weights. The data of 259 patients were used in this study and unpaired t-test was applied to the patient groups. The weight difference of the patient population was found as extremely significant $(\mathrm{p}<0.0001)$. The results demonstrated that the weight difference of female patient group was very significant $(\mathrm{p}=0.0012)$ and the male patient group was extremely significant $(\mathrm{p}<0.0001)$. The weight difference of young patients (18-34 years) was not significant $(0.7309)$, middle aged patients (35-64 years) was extremely significant $(\mathrm{p}<0.0001)$ and elderly patients (65 years and above) was not significant $(\mathrm{p}=0.3540)$. Therefore, weight change is prominent in most of the patients who are on heart transplantation waiting list. Employment of a dietitian or nutrition specialist to transplant multidisciplinary teams is advised, to minimize patient cachexia.
\end{abstract}

Keywords: Ideal body weight; Heart transplant; Nutrition; Heart failure; Transplantation; Cardiovascular

\section{Introduction}

Heart transplantation is a major operation that could be defined as a lifesaving procedure where the failing heart is replaced with a donor heart that is in a healthy state. It is highly reserved for patients whose condition cannot improve with medical or other surgical treatment methods. Numerous cardiological conditions such as cardiomyopathy, coronary artery disease, congenital heart defects, and disease of heart valves or even arrhythmias could lead to a requirement for heart transplant.

Possibility of transplanting a heart was first mentioned by Simon Flexener more than a decade ago, in the article he wrote in 1907 [1]. The first patient trial was performed as a xenotransplant in 1964, when James Hardy attempted to transplant the heart of a chimpanzee to a dying patient. The transplanted heart beated for over an hour before the patient's death $[2,3,4]$. The first allogenic human to human transplantation of heart was performed by Norman Shumway and Richard Lower in 1967. The operation was successful and the patient survived for 18 days before death that was caused by pneumonia [5,6]. Since then, heart transplantation operation has greatly improved and currently, it is applied globally in numerous centers on daily basis.

\footnotetext{
${ }^{*}$ Corresponding author: Ozlem Balcioglu

Department of Cardiovascular Surgery, Faculty of Medicine, Near East University, TRNC Mersin 10 Turkey.

Copyright (C) 2021 Author(s) retain the copyright of this article. This article is published under the terms of the Creative Commons Attribution Liscense 4.0.
} 
Although it is a commonly practiced operation, it is still considered as a major operation and there is a great range of complication possibilities for heart transplant patients such as infection, sepsis, graft rejection, arrhythmias, valve disorders, thrombosis or bleeding. As the number of hearts available for transplantation is very limited, rejection is a great concern and the risk of tissue rejection would never be eliminated for a recipient's lifetime. Because of this, recipients are required to use lifelong immunosuppressive medications and are continuously monitored [7,8].

As donor availability is very low and limited, a patient selection criteria has been set where the indication of presence of an end-stage heart disease that could not be treated by other measures is clearly defined: and, numerous contraindications such as having a major systemic disease, older than 70 years of age, presence of an active systemic or severe local infection, presence of a malignancy in the past 5 years (except localized skin cancers excluding melanoma) or recurrence possibility of previous malignancy, active cigarette or abusive substance usage, presence of a nonmanageable major psychiatric disorder or possession of severe neurological deficits $[9,10]$.

Improvements in technology and research has encouraged a quest for an alternative to heart transplantation operation which is limited by scarcity of donors. Ventricular assist devices (VAD) have been developed in last two decades from paracorporeal systems to intracorporeal systems; and, became an alternative treatment method for patients waiting in heart transplant list. Major indications of VADs are classified into four: bridge to transplantation (wait with VAD until receiving the eligible donor heart), destination therapy (candidates who have contraindications for transplant surgery, for example diabetes mellitus), and bridge to recovery (especially for acute heart failure) and bridge to decision (to keep the patient alive until making the most appropriate decision). Today, all VADs supply blood from left ventricle, through the aorta and are implanted to the patient's chest as intracorporeal [11].

As the average lifespan is increasing by advancements in medicine and technology, the number of people requiring a heart transplant is also increasing. Long waiting lists for transplantation operations is leading to worsening of patients' systemic health including weight changes. Increasing evidence suggests that preoperative weight, body mass index (BMI) and nutritional status of patients affect the transplant surgery outcomes [12,13]. However, there is very limited information about the preoperative state of patients in terms of weight.

\section{Material and methods}

Data of 259 patients aged 18 and above, who received heart transplants between 2000-2018 years, was used in this study.

Gender, age, height and weight of patients were recorded and ideal body weight (IBW) was calculated by the Lorentz formula [14]:

For male patients; IBW $=50+(0.91 \times[$ height in centimeters -152.4$])$

For female patients; IBW $=45.5+(0.91 \times[$ height in centimeters -152.4$])$

Of 259 patients, 56 were female and 203 were male. Moreover, 79 patients were young (18-34 years), 177 patients were middle aged (35-64 years old) and 3 patients were elderly (65 years old and older).

SPSS 21.0 (IBM, USA) software was used for the statistical analyses of this study. Unpaired t-test statistical analysis test ( $95 \%$ confidence interval, significance level of $\mathrm{p} \leq 0.05$ ) was applied to investigate the statistical significance of difference between weight and ideal body weight of patients.

The aim of this study was to test the statistical significance of the weight loss by using the actual weight and calculated IBW of heart transplant patients as the whole patient population, as different genders (male and female) and as different age groups (18-34 years: young, 35-64 years: middle aged, above 65 years and older: elderly). Patients below 18 years of age were excluded from this study.

\section{Results and discussion}

The age range of patients included in this study were 18 to 66 , with a mean of $42.54 \pm 12.90$ years. The height was differing from 145 to 197 centimeters and the mean height of patients was $169.37 \pm 9.43$ centimeters. The weight range of the study group was 40 to 110 kilograms, the mean weight was $69.46 \pm 13.56$ kilograms. The ideal body weight calculate for the patients was ranging from 47 to 85.3 kilograms, with a mean of $64.26 \pm 7.29$ kilograms. Descriptive 
statistics of 259 patients who were included in this study is demonstrated in Table 1 . The unpaired t-test result for comparison of weight and IBW difference of 259 patients was found to be extremely significant $(\mathrm{p}<0.0001)$.

Table 1 Descriptive statistics of the study group

\begin{tabular}{|l|c|c|c|c|}
\hline & Minimum & Maximum & Mean & Standard deviation \\
\hline Age (years) & 18.00 & 66.00 & 42.54 & 12.90 \\
\hline Height (centimeters) & 145.00 & 197.00 & 169.37 & 9.43 \\
\hline Weight (kilograms) & 40.00 & 110.00 & 69.46 & 13.56 \\
\hline IBW (kilograms) & 47.00 & 85.3 & 64.26 & 7.29 \\
\hline
\end{tabular}

The unpaired t-test analysis result of the weight and IBW difference was found to be statistically very significant $(p=0.0012)$ for 56 female patients; and, was found to be statistically extremely significant $(p<0.0001)$ for 203 male patients (Table 2). Therefore, it could be stated that female patients were found to be very thin and male patients were extremely thin when compared to their ideal body weights.

Table 2 Statistical analysis results of male and female patient groups

\begin{tabular}{|l|c|c|l|}
\hline Gender & Patient Population & Two-tailed p value & Unpaired t-test results \\
\hline Female & 56 & 0.0012 & Very significant \\
\hline Male & 203 & $<0.0001$ & Extremely significant \\
\hline
\end{tabular}

The results of unpaired t-test results were found to be statistically not significant $(\mathrm{p}=0.7309)$ for the young patient group (18-34 years old), extremely significant $(\mathrm{p}<0.0001)$ for the middle aged patient group (35-64 years old) and not significant ( $\mathrm{p}=0.3540)$ for the elderly patient group (65 years old and older). Therefore, it could be stated that when age-related analysis was investigated, weight of young and old patients were alike their ideal body weights whereas the middle aged patient group was extremely thinner than their ideal body weights.

Table 3 Statistical analysis results of different patient age groups

\begin{tabular}{|c|c|c|l|}
\hline Age range (years) & Patient Population & Two-tailed p value & Unpaired t-test results \\
\hline $18-34$ & 79 & 0.7309 & Not significant \\
\hline $35-64$ & 177 & $<0.0001$ & Extremely significant \\
\hline 65 and above & 3 & 0.3540 & Not significant \\
\hline
\end{tabular}

In the young patient group, a possible explanation for weight loss being not significant may be that these patients were more recently diagnosed and were on the waiting list for a shorter time. The non-significant weight alteration of elderly patient population could be as a result of a very low number of patients as age is a restriction for transplantation and alternatives such as VAD would be considered for this patient group.

Chronic heart failure is a systemic disease, with adverse effects on multiple organ systems. It is an irreversible disease with multi-organ manifestations. This creates both physiological and psychological manifestations on patients. Long term and severe chronic heart failure patients would suffer from both metabolic and psychological insufficiency and their eating habits change. Therefore, patients who are listed for heart transplantation surgery, lose weight continuously. However, sufficient attention has not been placed on this.

Recent research demonstrates there is a correlation between low body weight of patients and postoperative morbidity and mortality [15]. Moreover, it has been demonstrated that heart transplant patients gain weight postoperatively [16]. 
As the results of this study reveal, patients are significantly far from their ideal body weights prior to surgery and this situation could be set aright by employment of a dietician or nutrition specialist to the multidisciplinary transplant team.

\section{Conclusion}

This study demonstrates that there is an extreme difference the weights and IBWs of heart transplantation patient group. When gender-based differences were analyzed, female patients had a very different and male patients had an extremely different weight when compared to their IBWs. Moreover, in the age-based assessment, it was resoluted that young and elderly patients did not demonstrate a significant difference but the middle aged patient group demonstrated an extreme difference for their weight-IBW comparison.

As a result of this study, it would be advised that medical centers who perform heart transplantation surgery should employ a dietitian or a nutrition specialist to their multidisciplinary teams; and, a nutrition plan should be advised to the patients who are on heart transplantation waiting list, to attempt to minimize cachexia.

\section{Compliance with ethical standards}

\section{Acknowledgments}

We would like to thank Ege University Hospital Cardiovascular Surgery team for their efforts and dedication.

\section{Disclosure of conflict of interest}

The authors or this article have no conflicts of interest with any person, institution or company.

\section{Statement of ethical approval}

This study has received ethics committee approval.

\section{Statement of informed consent}

Informed consent for use of data in research, was obtained from all individual participants included in the study.

\section{References}

[1] https://timesmachine.nytimes.com/timesmachine/1908/01/02/104713376.pdf The New York Times, January 2, 1908.

[2] Hardy James D, Chavez Carlos M, Kurrus Fred D, Neely William A, Eraslan Sadan, Turner M Don, Fabian Leonard W, Labecki Thaddeus D. "Heart Transplantation in Man". JAMA. 1964; 188(13).

[3] Jump up to:a b Every Second Counts: The Race to Transplant the First Human Heart, Donald McRae, New York: Penguin (Berkley/Putnam), Ch. 7 "Mississippi Gambling". This source states the heartbeat for approximately one hour. 2006; 123-27.

[4] James D. Hardy, 84, Dies; Paved Way for Transplants, Obituary, New York Times (Associated Press), Feb. 21, 2003. This source states the transplanted chimpanzee heartbeat for 90 minutes.

[5] McRae D. Every Second Counts: The Race to Transplant the First Human Heart, New York: Penguin (Berkley/Putnam). 2006.

[6] Norman Shumway. Father of heart transplantation who also performed the world's first heart-lung transplant, Obituary, The Independent [UK], 16 Feb. 2006.

[7] Bishay R. The 'Mighty Mouse' Model in Experimental Cardiac Transplantation. Hypothesis. 2011; 9(1): e5.

[8] Costanzo MR, Dipchand A, Starling R, Anderson A, Chan M, Desai S, Fedson S, Fisher P, et al. The International Society of Heart and Lung Transplantation Guidelines for the care of heart transplant recipients. The Journal of Heart and Lung Transplantation. 2010; 29(8): 914-56.

[9] https://www.hopkinsmedicine.org/transplant/referring-physicians/heart-transplant-criteria.html

[10] de Jonge, $\mathrm{N}$ et al. "Guidelines for heart transplantation." Netherlands heart journal: monthly journal of the Netherlands Society of Cardiology and the Netherlands Heart Foundation. 2008; 16(3): 79-87. 
[11] https://www.mayoclinic.org/tests-procedures/ventricular-assist-device/about/pac-20384529

[12] https://pubmed.ncbi.nlm.nih.gov/19940761/

[13] https://europepmc.org/article/med/8889981

[14] https://pubs.asahq.org/anesthesiology/article/127/1/203/18747/Calculating-Ideal-Body-Weight-Keep-ItSimple

[15] https://pubmed.ncbi.nlm.nih.gov/19940761/

[16] https://pubmed.ncbi.nlm.nih.gov/16399528/ 